Individual Participant Data Analysis of two Trials on Aldosterone Blockade in

\title{
Myocardial Infarction
}

Farzin Beygui ${ }^{1}$, Eric Van Belle ${ }^{2}$, Patrick Ecollan ${ }^{3}$ Jacques Machecourt $^{4}$, Christian W.

Hamm $^{5}$, Esteban Lopez De Sa ${ }^{6}$, Marcus Flather ${ }^{7}$, Freek Verheugt ${ }^{8}$, Eric Vicaut ${ }^{9}$, Faiez

Zannad $^{10}$, Bertram Pitt1 ${ }^{11}$, Gilles Montalescot ${ }^{12}$.

Corresponding author : Pr. Farzin Beygui, ACTION Study Group, Service de Cardiologie, Centre Hospitalier Universitaire de Caen, EA4650 Normandie Université, France. beygui-f@ chu-caen.fr; Tel: +33 2 31063350

Journal: Heart

Word count : 3033 (max. 3000) Abstract: 249 (max. 250) Figures: 2

Tables: 2 References: $30 \quad$ Online supplementary tables: 3 Online

supplementary figure: 1 


\section{Abstract \\ Background}

Two recent randomized trials studied the benefit of Mineralocorticoid receptor antagonists (MRA) in ST-elevation myocardial infarction (STEMI) irrespective or in absence of heart failure. The studies were both undersized to assess hard clinical endpoints. A pooled analysis was pre-planned by the steering committees.

\section{Methods}

We conducted a pre-specified meta-analysis of patient-level data of STEMI patients recruited in 2 multicenter superiority trials, randomized within 72 hours after symptom onset. Patients were allocated (1:1) to 2 MRA regimens: 1 . An intravenous bolus of potassium canrenoate (200 mg) followed by oral spironolactone (25 mg od) versus standard therapy; or 2. Oral Eplerenone (25 to $50 \mathrm{mg}$ ) in versus placebo. The primary and key secondary outcomes, all cause death and the composite of all cause death or resuscitated sudden death respectively, were assessed in the intention to treat population using a Cox model stratified on the study identifier.

\section{Results}

Patients were randomly assigned to receive $(n=1118)$ or not the MRA regimen $(n=1123)$. After a median follow-up time of 188 days, the primary and secondary outcomes occurred in $5(0.4 \%)$ and $17(1.5 \%)$ patients (adjHR $0.31,95 \% \mathrm{CI} 0.11$ to $0.86, \mathrm{p}=0.03)$ and, $6(0.5 \%)$ and 22(2\%) patients (adjHR 0.26; 95\% CI 0.10-0.65, $\mathrm{p}=0.004)$ in the MRA and control groups respectively. There were also trends toward lower rates of cardiovascular death $(\mathrm{p}=0.06)$ and ventricular fibrillation $(\mathrm{p}=0.08)$ in the MRA group.

\section{Conclusion}

Our analysis suggests that compared to standard therapy MRA regimens are associated with a reduction of death and death or resuscitated sudden death in STEMI. 


\section{What is already known about this subject?}

Mineralocorticoid receptor antagonists reduce mortality in the setting of myocardial infarction complicated by heart failure.

\section{What does this study add?}

The study shows that regardless of heart failure, mineralocorticoid receptor antagonists reduce mortality in the setting of ST elevation myocardial infarction, in addition to optimal treatment.

\section{How might this impact on clinical practice?}

The use of very low cost mineralocorticoid receptor antagonists may be considered in ST elevation myocardial infarction patients irrespective of heart failure to reduce mortality 


\section{Introduction}

\section{Rationale}

High aldosterone plasma levels early after myocardial infarction are associated with mortality, sudden cardiac death and heart failure. [1-5] Experimental studies have shown that early administration of MRA after myocardial infarction could improve myocardial healing and both electrical and structural remodeling.[6,7] Small-sized studies have also reported a benefit of MRAs, initiated early after myocardial infarction in the prevention of left ventricular remodeling [8,9] and life-threatening arrhythmia.[10,11]

Mineralocortoid receptor antagonists (MRA) spironolactone and eplerenone reduce mortality in the setting of congestive heart failure with reduced ejection fraction and myocardial infarction complicated by left ventricular dysfunction and heart failure.[12-14] The Double-Blind, Randomized, Placebo-Controlled Trial Evaluating The Safety And Efficacy Of Early Treatment With Eplerenone In Patients With Acute Myocardial Infarction (REMINDER) [15] showed that eplerenone used within the first 24 hours of ST-segment elevation MI (STEMI) excluding patients with heart failure, was safe and effective on a composite outcome mainly driven by the biological outcome of a lower plasma level of Btype natriuretic peptide. More recently the Aldosterone Lethal effects Blockade in Acute myocardial infarction Treated with or without Reperfusion to improve Outcome and Survival at Six months follow-up (ALBATROSS) failed to demonstrate a benefit of a spironolactonebased MRA regimen in improving outcome in the general setting of STEMI or non-STEMI irrespective of heart failure, but suggested a potential significant reduction of rates of death by the MRA in the STEMI subgroup of patients (77\% of the population).[16] Both ALBATROSS and REMINDER studies were undersized to detect a difference in rates of hard clinical outcomes. A pooled analysis of individual participant-level data was preplanned by the steering committees of the 2 trials prior to the end of the inclusion periods. 


\section{Objectives}

The aim of this pooled analysis of patient-level data was to investigate the effect of a MRA regimen initiated early after the onset of STEMI, irrespective or in absence of heart failure, on mortality and the composite of death or resuscitated sudden death.

\section{Methods}

\section{Study design and population}

Our study used pooled individual patient-level data of the STEMI subgroup of the ALBATROSS[16] and the total population of the REMINDER[15] randomized trials. Both trials studied the efficacy of an MRA regimen in addition to standard therapy in comparison with either standard therapy alone or associated with a placebo. The designs of the studies are briefly depicted in supplementary table 1. ALBATROSS- and REMINDER-eligible patients were men and non-pregnant women presenting with STEMI within 72 and 24 hours of symptom onset respectively. REMINDER included STEMI patients only while ALBATROSS included not only STEMI ( $\mathrm{n}=1229)$ but also Non-STEMI patients $(\mathrm{n}=369)$ with a significant interaction between the presentation type and treatment with respect to mortality. Hence only STEMI patients of the ALBATROSS trial were included in the present analysis. Other differences between the ALBATROSS and REMINDER studies included the treatment (IV potassium canrenoate followed by oral spironolactone versus oral eplerenone), the timing of the randomization with respect to symptom onset (within 24 versus within 72 hours) and the duration of follow-up (182 \pm 40 versus $317 \pm 218$ days). Furthermore, to be included in REMINDER no sign of heart failure was to be present at randomization while in ALBATROSS patients were included regardless of signs of heart failure. The comparison between the patients' characteristics of the 2 studies is reported in supplementary table 2 .

\section{Outcomes}


The primary outcome of the ALBATROSS trial was the composite of death, resuscitated cardiac arrest, significant ventricular arrhythmia, class IA indication[17] for implantable defibrillator, new or worsening heart failure over the period of 6 months following randomization. The primary outcome of the REMINDER trial was the composite of cardiovascular mortality, re-hospitalization or extended initial hospital stay for heart failure or sustained ventricular tachycardia or ventricular fibrillation, left ventricular ejection fraction $\leq$ $40 \% \geq 1$ month post- randomization or high BNP or NT-proBNP levels.[15]

The primary efficacy outcome of the present study was mortality of any cause. As BNP was not measured in ALBATROSS and there were differences between definitions of events between the trials such as heart failure (requiring any treatment versus hospitalization or emergency room visit) or sustained/significant ventricular tachycardia (requiring any treatment versus hospitalization), any endpoint other than mortality was considered as a secondary endpoint. The key secondary efficacy outcome was the composite of death and resuscitated sudden death. Other secondary efficacy outcomes analyzed included cardiovascular death, resuscitated cardiac death, ventricular fibrillation, any ventricular arrhythmia (ventricular tachycardia or fibrillation), stroke, implanted cardiac defibrillator, class IA[17] indication for defibrillators-whether implanted or not- and recurrent myocardial infarction -study specific definitions-. An independent committee adjudicated all outcomes in both trials.

Safety outcomes included Hyperkalemia $>5.5$ and $>6 \mathrm{mmol} / \mathrm{L}$ at any time after randomization and acute renal failure defined by an increase in estimated glomerular filtration rate (eGFR) of $\geq 25 \%$ in comparison with admission eGFR. For the present analysis, eGFR was calculated for all patients based on Cockroft-Gault method.

\section{Statistical analysis}

All analyses used individual patient-level data of the pooled STEMI subgroup of the 
ALBATROSS and the total population of the REMINDER trials. The pooled analysis of the two patient populations was pre-planned by the steering committees of the two trials. The full raw datasets were provided by the studies' organizers and analyzed independently from the published data. All data were assessed within the 2 sets for completeness and coherence. No specific issue was observed.

A one stage approach was used for the analysis. Data were compared between treatment arms using the Cochran-Mantel-Haenszel test and a 2-way ANOVA both using the study identifier as the stratification variable. $\chi^{2}$ test and Student's $t$ test were used for the comparison of the variables between the studies.

All efficacy endpoints were analyzed as time-to-event outcomes using Cox models systematically stratified on the study identifier with a follow-up time of 188 days equal to the median follow-up time of the pooled data. Kaplan-Meier cumulative hazards curves were drawn for the comparison of the primary and key secondary outcomes between treatment groups. Safety endpoints were analyzed as binary variables compared between treatment groups using a Cochran-Mantel-Haenszel test stratified on the study identifier.

Post hoc sensitivity analyses were performed for all efficacy endpoints using the Cox models stratified on the study identifier and adjusted on variables unequally distributed between the treatment arms with a p value threshold of $<0.20$.

Inter-study heterogeneity assessments were performed by the measurement of the interaction between the study identifier and the treatment arm using a cox model for efficacy endpoints and a logistic regression model for safety endpoints without stratification.

Interaction between subgroups pre-defined in the two trials and treatment was tested using the Cox models stratified on the study identifier when data were available for both trials. 
All tests had a two-sided significance level of 5\% and were performed using R software, version 3.2.3 for Mac ( $\mathrm{R}$ foundation for statistical computation).

\section{Results}

A total of 2241 patients were randomly assigned to the MRA regimen (1118 patients) or control regimen (1123 patients).

Despite the differences between ALBATROSS and REMINDER trials (supplementary table 1), the active and control groups were well balanced with respect to most baseline characteristics and treatment strategies (Table 1) with the exception of significantly higher heart rate $(\mathrm{p}=0.02)$ and systolic blood pressure $(\mathrm{p}=0.02)$, as well as higher rates of Killip class

$\geq$ II on admission in the control group $(\mathrm{p}=0.02)$. The standard of care for the index event included fibrinolysis in $189(8.4 \%)$, primary percutaneous coronary intervention (PCI) in $1954(87.2 \%)$, any PCI in 2060 (91.9\%) and coronary artery bypass surgery in $41(1.8 \%)$ patients. 
Table 1. Patient characteristics according to treatment

\begin{tabular}{|c|c|c|c|c|}
\hline Variable & $\begin{array}{l}\text { Overall } \\
n=2241\end{array}$ & $\begin{array}{l}\text { Active } \\
n=1118\end{array}$ & $\begin{array}{l}\text { Control } \\
n=1123\end{array}$ & $p$ \\
\hline Age (years) & $58 \pm 12$ & $58 \pm 11$ & $58 \pm 12$ & 0.8 \\
\hline Gender (Female) & $377(16.8 \%)$ & $178(15.9)$ & 199(17.7) & 0.3 \\
\hline \multicolumn{5}{|l|}{ Admission characteristics } \\
\hline Body mass index $\left(\mathrm{Kg} / \mathrm{m}^{2}\right)$ & $27 \pm 5$ & $27 \pm 5$ & $27 \pm 5$ & 0.12 \\
\hline Heart rate (per minute) & $73 \pm 15$ & $73 \pm 15$ & $74 \pm 15$ & 0.02 \\
\hline \multicolumn{5}{|l|}{ Systolic blood } \\
\hline pressure $(\mathrm{mmHg})$ & $124 \pm 21$ & $123 \pm 21$ & $125 \pm 21$ & 0.02 \\
\hline Killip class & $1.05 \pm 0.28$ & $1.03 \pm 0.22$ & $1.06 \pm 0.33$ & 0.007 \\
\hline Killip class $\geq 2$ & $82(3.7)$ & $29(2.6)$ & $53(4.7)$ & 0.007 \\
\hline Plasma creatinine $(\mu \mathrm{mol} / \mathrm{L})$ & $81.8 \pm 22.3$ & $82 \pm 22$ & $81 \pm 23$ & 0.4 \\
\hline eGFR (mL/min) & $104 \pm 36$ & $102 \pm 35$ & $105 \pm 37$ & 0.14 \\
\hline Plasma potassium (mmol/L) & $4.02 \pm 0.5$ & $4.03 \pm 0.5$ & $4.01 \pm 0.4$ & 0.3 \\
\hline GRACE score & $140 \pm 23$ & $140 \pm 22$ & $140 \pm 24$ & 0.6 \\
\hline
\end{tabular}

Medical history

$\begin{array}{lcccr}\text { Systemic hypertension } & 964(43.0) & 464(41.5) & 500(44.5) & 0.15 \\ \text { Diabetes mellitus } & 298(13.3) & 145(13.0) & 153(13.6) & 0.7 \\ \text { Hypercholesterolemia } & 883(39.4) & 450(40.3) & 433(38.6) & 0.4 \\ \text { Active smoking } & 772(34.5) & 375(33.5) & 397(35.4) & 0.4 \\ \text { Prior myocardial infarction } & 136(6.1) & 71(6.4) & 65(5.8) & 0.6 \\ \text { Prior stroke } & 44(2.0) & 28(2.5) & 16(1.4) & 0.09\end{array}$


Initial/in-hospital management

\begin{tabular}{|c|c|c|c|c|}
\hline Primary $\mathrm{PCl}$ & 1954(87.2) & $971(86.9)$ & $983(87.5)$ & 0.7 \\
\hline Any $\mathrm{PCl}$ & 2060(91.9) & 1028(91.9) & 1032(91.9) & 0.96 \\
\hline Coronary Bypass surgery & $41(1.8)$ & $23(2.1)$ & $18(1.6)$ & 0.5 \\
\hline Fibrinolysis & $189(8.4)$ & $96(8.6)$ & $93(8.3)$ & 0.8 \\
\hline Glycoprotein IIBIIIA inhibitors & $833(37.2)$ & $409(36.6)$ & $424(37.8)$ & 0.6 \\
\hline Aspirin & 2201(98.2) & 1096(98.0) & 1105(98.4) & 0.6 \\
\hline Clopidogrel & 1445(64.5) & $734(65.7)$ & $711(63.3)$ & 0.3 \\
\hline Prasugrel & $1273(56.8)$ & $619(55.4)$ & $654(58.2)$ & 0.2 \\
\hline Ticagrelor & $28(1.3)$ & $13(1.2)$ & $15(1.3)$ & 0.9 \\
\hline Any $\mathrm{p} 2 \mathrm{y} 12$ inhibitor & 2225(99.3) & 1110(99.3) & 1115(99.3) & 1.0 \\
\hline ACEi or ARB & 1964(87.6) & $975(87.2)$ & $989(88.1)$ & 0.6 \\
\hline Beta-blockers & 2077(92.7) & $1037(92.8)$ & 1040(92.6) & 0.95 \\
\hline Diuretics & 293(13.1) & $135(12.1)$ & $158(14.1)$ & 0.2 \\
\hline Statins & 2171(96.9) & $1076(96.2)$ & 1095(97.5) & 0.11 \\
\hline LVEF* & $53 \pm 9$ & $53 \pm 9$ & $53 \pm 9$ & 0.2 \\
\hline ollow-up duration (days) & $243 \pm 164$ & $244 \pm 165$ & $241 \pm 163$ & 0.7 \\
\hline \multicolumn{5}{|c|}{ ata are $n(\%)$ or mean \pm SD. LVEF : left ventricular ejection fraction (*available in 2014} \\
\hline
\end{tabular}


As reported in table 2, after a median follow-up of 188 days (interquartile range 52 days), the primary outcome (Figure 1) occurred in $5(0.4 \%)$ and $17(1.5 \%)$ patients in the active and control groups, respectively (HR 0.30, 95\% CI 0.11-0.81); $\mathrm{p}=0.01$ and adjusted-HR 0.31, 95\% CI 0.11-0.86; $\mathrm{p}=0.03$ ). Two un-adjudicated deaths reported as security events 389 and 528 days after randomization in the control and the active groups of the REMINDER trial respectively were not included in the analysis. All resuscitated sudden deaths were reported within the analysis time. The key secondary outcome of death or resuscitated sudden death (Figure 2) occurred in $6(0.5 \%)$ and $22(2 \%)$ patients in the active and control groups, respectively (HR 0.27, 95\% CI 0.11-0.67; $\mathrm{p}=0.002$ and adjusted-HR 0.26, 95\% CI 0.10-0.65; $\mathrm{p}=0.004)$. There were also trends toward lower rates of death from cardiovascular causes $(\mathrm{p}=0.06)$ and ventricular fibrillation $(\mathrm{p}=0.08$ and adjusted-HR $)$ in the active group. Other assessed efficacy endpoints did not differ between the two groups. The two trials were different with respect to the rates of the primary and secondary key outcomes as well as several other outcomes (supplementary table 3). However with the exception of a trend $(p=0.06)$ towards a heterogeneity in rates of recurrent myocardial infarction, no significant inter-study heterogeneity was detected. 


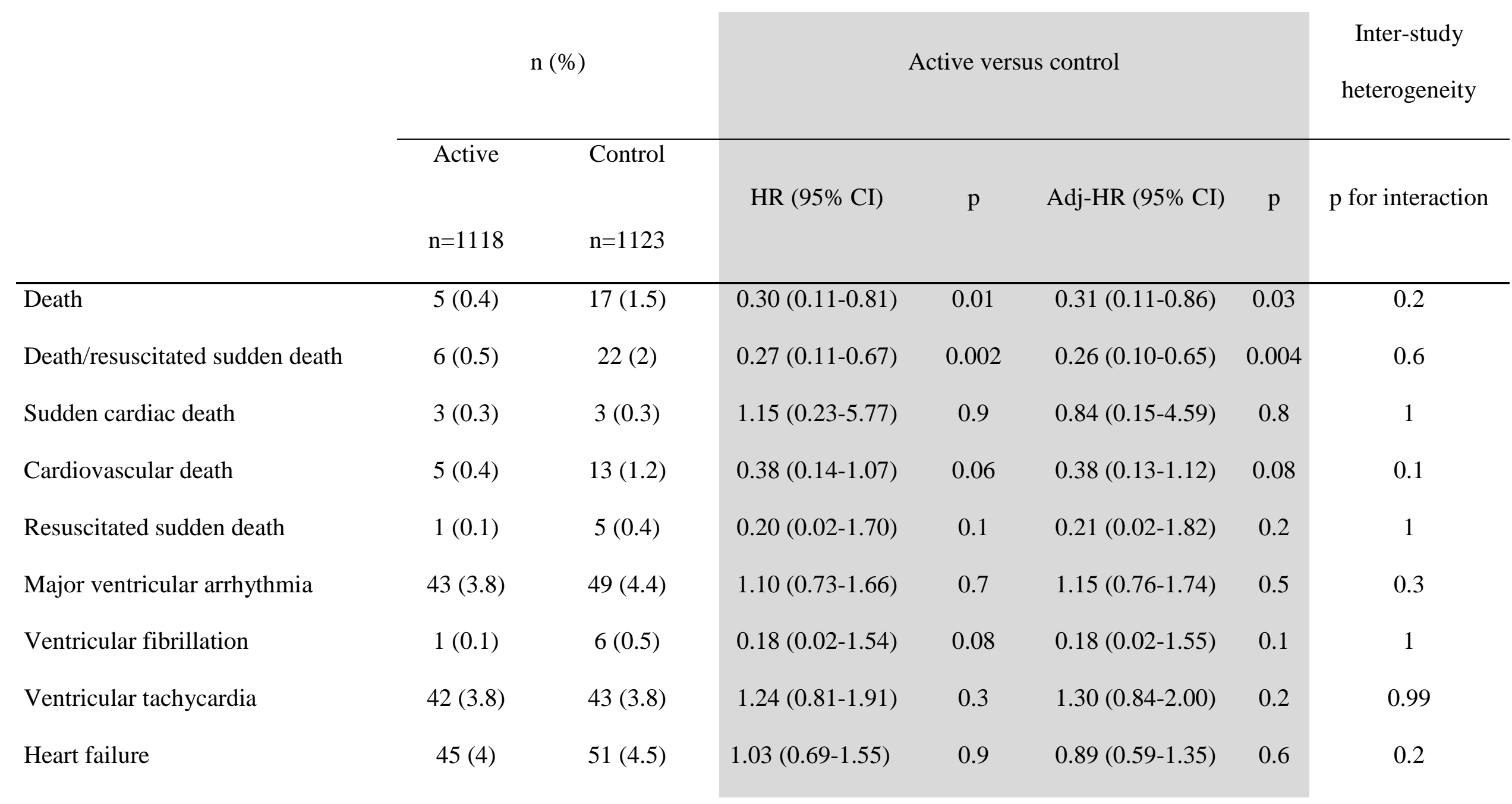




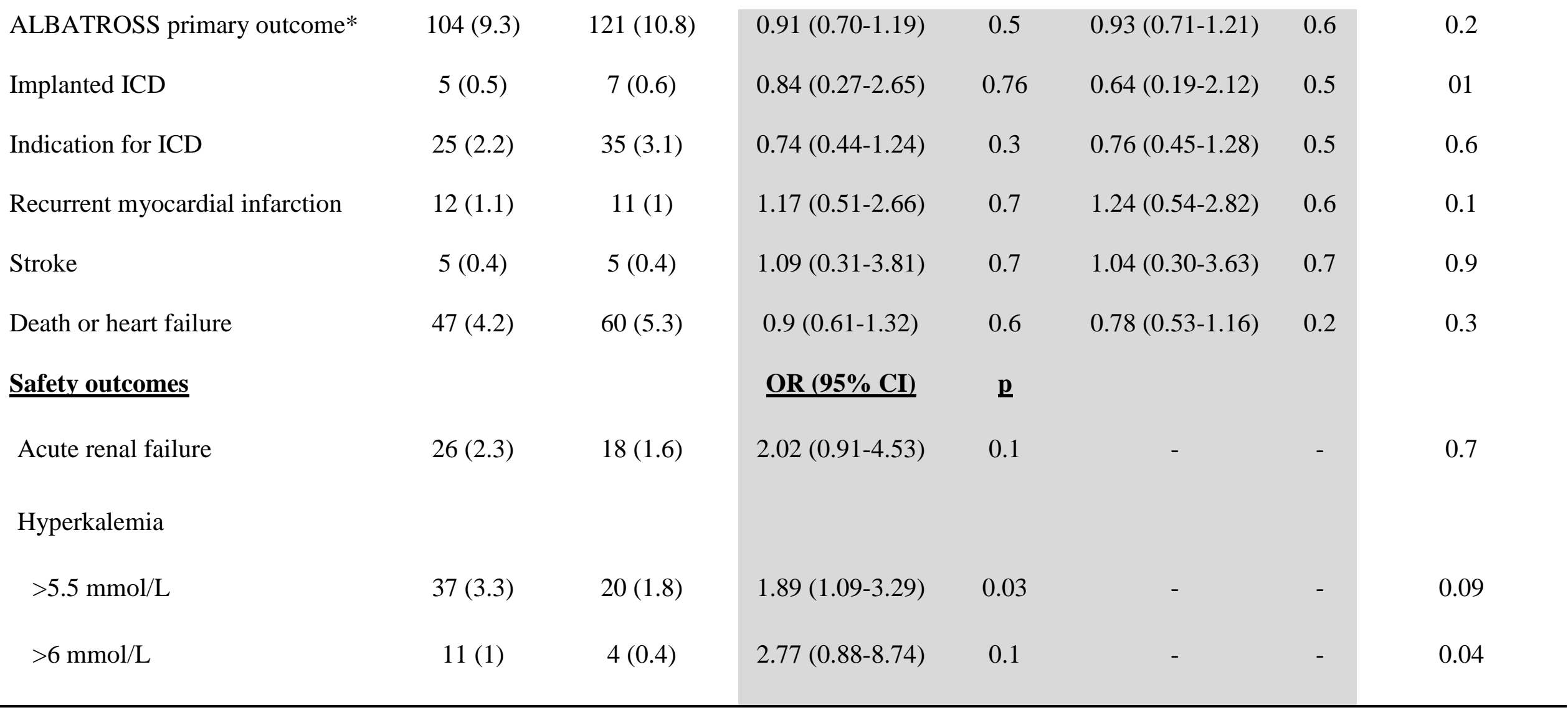

*The composite of death, resuscitated sudden death, ventricular arrhythmia, heart failure and indication for ICD. Adj-HR: HR adjusted on variable unequally distributed between treatment arms with a threshold $\mathrm{p}$ value $<0.2-$ i.e. baseline binary killip class, heart rate, systolic blood pressure, eGFR, body mass index and, history of systemic hypertension and stroke. 
The results were consistent among all subgroups with respect to the primary and secondary key outcomes (supplemantry Figure). HR could not be calculated in the patient subgroups with a Killip class $\geq 2$ in the active arms, as well as all patients with a grace score $<109$ because of absence of any primary or secondary key outcome. HR could also not be calculated for death in patients $<65$ because of absence of event in the active arm.

Hyperkalemia $>5.5 \mathrm{mmol} / \mathrm{L}$ occurred in $37(3.3 \%)$ and $20(1.8 \%)$ patients in the active and control groups, respectively (stratified OR $1.89 ; 95 \% \mathrm{CI}, 1.09$ to $3.29, \mathrm{p}=0.03$ ) while rates of Hyperkalemia > $6 \mathrm{mmol} / \mathrm{L}$ and acute renal failure were not significantly different between the two groups. A trend toward $(\mathrm{p}=0.09)$ and a significant inter-study heterogeneity $(\mathrm{p}=0.04)$ were detected with respect to the hyperkalemia $>5.5$ and 6 outcomes.

\section{Discussion}

Our analysis based on the pooled patient-level data of the two recent randomized trials addressing the question of early MRA blockade in low risk STEMI shows reduced rates of death compared to standard therapy. Although the subgroup analysis should be considered as only exploratory, it suggests a consistent effect of the treatment. MRA blockade appears to be associated with reduced risks of allcause mortality and the composite of death or resuscitated sudden cardiac death. However the analysis failed to show a benefit with respect to other endpoints including heart failure or ventricular arrhythmia. Rates of hyperkalemia $>5.5 \mathrm{mmol} / \mathrm{L}$ were significantly higher among patients treated with MRA.

Experimental studies have shown that the early use of MRA after MI reduces left ventricular expansion and extensive fibrosis by antagonizing activation of the mineralocorticoid receptor by aldosterone and cortisol.[18-20] The clinical benefit of MRA have been extensively demonstrated in heart failure irrespective of its ischemic or non-ischemic origin. [12,13] MRA use is also associated with a reduction of mortality in patients with heart failure and reduced systolic ventricular function 
after myocardial infarction.[14] Thus, the addition of MRAs to beta-blockers and angiotensin converting enzyme inhibitors has been highly recommended [21-23] in patients with heart failure and reduced left ventricular function irrespective of the etiology of heart failure.

In the Eplerenone Post Acute MI Heart Failure Efficacy and Survival Study (EPHESUS) ${ }^{15}$ eplerenone was initiated 3 to 14 days after the onset of MI complicated by heart failure and reduced left ventricular function. The benefit observed was apparently driven by the group of patients treated earlier (i.e. 3-7 days).[24] This finding is consistent with other studies reporting high aldosterone plasma levels early after MI[25,26] and the relationship of these levels with clinical outcomes.[1-3] The REMINDER study recruited low risk STEMI patients with no heart failure at admission and very low mortality rates. Although REMINDER showed a benefit of eplerenone over placebo, the benefit was driven by the B-type natriuretic peptide levels with only non-significant trends on clinical outcomes.[15] The ALBATROSS trial recruited a broader STEMI population at higher risk as compared with REMINDER. ALBATROSS STEMI population included all-comers presenting for primary PCI, few with heart failure (6.7\%), with significantly higher admission GRACE scores but also more contemporary and optimal treatment as compared to REMINDER. Although the study failed to show the benefit of MRAs on the primary outcome it highlighted a significant signal in favor of a MRA effect on mortality in STEMI. The present pooled analysis includes a relatively young and lowto-intermediate risk STEMI population (mean grace score 140) treated optimally with very high rates of revascularization and optimal medical therapy. The mortality reduction with MRAs in addition to an optimal management in such a low risk population should not be neglected. The previously mentioned pre-clinical and clinical data, the reduction of mortality in the setting of chronic heart failure or post myocardial infarction heart failure as well as the strength of the association support the plausibility of a MRA effect on mortality in the setting of STEMI. The early blunting of biological effects of MR activation after acute coronary artery occlusion[7,6,19], may lead to a favorable effect in STEMI 
through the un-triggering of the neurohormonal activation and the subsequent post-MI fibrosis and remodeling both leading to higher risk of death.

A recently published study-level meta-analysis of eleven randomized trials showed also the benefit of MRAs on mortality [27]. The study included STEMI and non-STEMI patients, with heart failure as an inclusion criteria or not. However the benefit of the MRAs was driven by studies that included patients with heart failure. Compared to the latter study, our patient-level analysis pooling only STEMI patients, without heart failure as an inclusion criteria and with a more adequate univariable and adjusted analysis of censored data may be considered as more sensitive in detecting differences between groups.

Interestingly, the MRA-related reduction of mortality found in our study was not associated with a concordant significant reduction of major ventricular arrhythmia or heart failure, although both events occurred numerically less in the active treatment group.

Sustained ventricular tachycardia or fibrillation have been reported to occur in $5.2 \%$ of patients after primary PCI essentially in the 48 first hours in the relatively recent analysis of the Harmonizing Outcomes with RevasculariZatiON and Stents in Acute Myocardial Infarction (Horizons-AMI) randomized trial.[28] In the latter study, such events did not portend decreased survival. The rates of ventricular tachycardia or fibrillation were approximately $4 \%$ in the pooled population of our analysis, with a difference between rates in the ALBATROSS (7.2\%) and the REMINDER trials (0.4\%). Such a difference is likely to be due to the difference of the definitions of ventricular tachycardia (requiring hospitalization) in REMINDER but also due to a more selected patient population without heart failure at admission, with preserved ejection fraction and with extremely low event rates - i.e death, heart failure, indication for ICD-. The absence of effect on rates of ventricular arrhythmia in our study is not in contradiction with a possible MRA effect on mortality. Concordant with our study, in both Randomized Aldosterone Evaluation Study (RALES)[12] and EPHESUS[14] trials mortality was reduced with MRA despite the absence of effect on the rates of ventricular arrhythmia. Moreover, in 
concordance with the above-mentioned study[28] most ventricular tachycardia were reported within only 48hours after randomization and such events may not impact mortality. Finally ventricular arrhythmia suppression after myocardial infarction is known not to improve survival.[29] Considering the trend towards a reduction of rates of ventricular fibrillation but not ventricular tachycardia in our analysis, it is plausible that MRAs may reduce the rates of lethal arrhythmia, or prevent the fatal issue of arrhythmia without affecting the frequency of post-STEMI arrhythmia in general.

The global rate of hospitalization for heart failure (4.4\%) in a recent multicenter real-life cohort of STEMI patients undergoing primary PCI [30] was higher than in REMINDER (1.4\%) but lower than in ALBATROSS (6.3\%). The differences in study definitions, the low-risk populations and the short durations of follow-up could explain the absence of detectable MRA effect on heart failure in the current analysis.

Our analysis also highlights the relative safety of the MRA regimens used in the studies. Although the rates of hyperkalemia $>5.5 \mathrm{mmol} / \mathrm{L}$ were higher in the MRA group than in the control group, they remained lower than what has been previously reported[14,15] and the rates of hyperkalemia $>6$ $\mathrm{mmol} / \mathrm{L}$ and acute renal failure were not different between treatment groups. However the results on hyperkalemia should be considered with great precaution because of a significant inter-study heterogeneity.

Our analysis has limitations inherent to its design pooling data from different studies with different designs and different active treatments. However a class effect is very likely between the drugs and the stratified and adjusted analyses as well as absence of detected inter-study heterogeneity may minimize the risk of bias. Furthermore event rates remained low and event definitions were different for ventricular tachycardia and heart failure. Nevertheless with respect to the primary and the key secondary outcomes of our study -death and death or resuscitated sudden death- the analysis may be considered as robust. The adjusted analysis should be interpreted with the consideration of low event rates, especially with respect to mortality. However the similar magnitude of effects and their 
confidence intervals between the un-adjusted and adjusted analyses supports the robustness of the findings. The subgroup analysis should be considered with great precaution because of the risk of multiple testing with low event rates. Whether the benefit of MRA may interact with the infarct location or the culprit vessel could not be assessed by our study because of lack of data.

Although the present report is the largest experience in STEMI outside of the scope of heart failure, it may lack power to detect differences between several studied outcomes. Larger and specifically designed studies are needed to assess such outcomes.

\section{Conclusions}

Our analysis based on pooled patient-level data from two randomized trials suggests a significant reduction of death and death or resuscitated sudden death associated with MRA regimens given early after low risk STEMI. Despite strong recommendations based on robust clinical evidence in the setting of heart failure, MRAs are still underused in routine clinical practice. Our data on both efficacy and safety of MRAs may contribute to extend the use of these life-saving and low-cost drugs. Although our study seems robust with respect to its primary outcome, the low event rates highlight the need for more studies adequately sized and specifically designed to confirm the potentially major clinical benefit associated with low-cost treatments. 


\section{Glossary}

ALBATROSS : Aldosterone Lethal effects Blockade in Acute myocardial infarction Treated with or without Reperfusion to improve Outcome and Survival at Six months follow-up. Clinical trial registration: NCT01176968

REMINDER : A Double-Blind, Randomized, Placebo-Controlled Trial Evaluating The Safety And Efficacy Of Early Treatment With Eplerenone In Patients With Acute Myocardial Infarction. Clinical trial registration:NCT01176968

\section{Author affiliations}

1. ACTION Study Group, Service de Cardiologie, Centre Hospitalier Universitaire de Caen, EA4650 Normandie Université, France; 2. INSERM U1011 et Service de Cardiologie, Institut Coeur Poumon, CHRU de Lille, France; 3. ACTION Study Groupe, SAMU, Centre Hospitalier Universitaire PitiéSalpêtrière, France; 4. Service de Cardiologie, Centre Hospitalier Universitaire de Grenoble, France; 5. Kerckhoff Clinic, Bad Nauheim, Germany; 6. University Hospital La Paz, Madrid, Spain; 7. University of East Anglia and Norfolk and Norwich University Hospital, Norwich, United Kingdom; 8. Hospital Onze Lieve Vrouwe Gasthuis, Amsterdam, Netherlands; 9. ACTION Study Group, Unite de Recherche Clinique, Hôpital Lariboisière, Paris, France; 10. Inserm, CIC 1433 et Pôle de Cardiologie, Centre Hospitalier Universitaire de Nancy, France;11. University of Michigan Hospital, Ann Arbor, United States of America; 12. Sorbonne Université-Paris 6, ACTION Study Group, Institut de Cardiologie (AP-HP), Centre Hospitalier Universitaire Pitié-Salpêtrière, INSERM UMRS 1166, Paris, France

\section{Sources of funding}

The ALBATROSS trial was led by members of the non-profit academic research organization ACTION, based at Pitié-Salpêtrière Hospital, Paris, France (www.action-coeur.org). The study was sponsored by the Assistance Publique-Hôpitaux de Paris (AP-HP) and exclusively funded by public 
grants from the French Ministry of Health and the Foundation of the Institute of Cardiometabolism And Nutrition (ICAN).

The REMINDER trial was funded by Pfizer. The REMINDER steering committee and Pfizer provided the data and gave consent for the pooled analysis.

The funding organizations had no involvement in the analysis of the results or writing of the present manuscript. No specific funding was used to support this work.

\section{Contributors}

Study concept and design: FB, EV, GM. Analysis and interpretation of data: FB, EV. Drafting of the manuscript: FB, GM. Critical revision of the manuscript for important intellectual content: EVB, PE, JM, CWH, ELDS, MF, FV, FZ, BP. Statistical analysis: FB, EV. Study supervision/guarantor: GM, BP.

The first and last author had full access to all the data, prepared the first draft of the manuscript. The manuscript was approved by all co-authors, who made the decision to submit the manuscript for publication and assumed responsibility for data accuracy.

The Corresponding Author has the right to grant on behalf of all authors and does grant on behalf of all authors, an exclusive license on a worldwide basis to the BMJ Publishing Group Ltd and its Licensees to permit this article to be published in HEART editions and any other BMJPGL products to exploit all subsidiary rights

\section{Competing interests}

F Beygui has received institutional grants and/or lecture or consultancy honoraria from AstraZeneca, Medtronic, Boston Scientific, Biosensor and Acist.

C Hamm has received speaker fees from Pfizer

G Montalescot declares his competing interests on http://action-coeur.org/declarations-dinterets/

\section{Ethics approval}


The REMINDER trial was approved by each participating centre's ethics committee; the ALBATROSS trial was approved by the French National Institutional Ethical Review Board. 


\section{References}

1 Beygui F, Collet J-P, Benoliel J-J, et al. High plasma aldosterone levels on admission are associated with death in patients presenting with acute ST-elevation myocardial infarction. Circulation 2006;114:2604-10. doi:10.1161/CIRCULATIONAHA.106.634626

2 Palmer BR, Pilbrow AP, Frampton CM, et al. Plasma aldosterone levels during hospitalization are predictive of survival post-myocardial infarction. Eur Heart J 2008;29:248996. doi:10.1093/eurheartj/ehn383

3 Beygui F, Montalescot G, Vicaut E, et al. Aldosterone and long-term outcome after myocardial infarction: A substudy of the french nationwide Observatoire sur la Prise en charge hospitalière, l'Evolution à un an et les caRactéristiques de patients présentant un infArctus du myocarde avec ou sans onde Q (OPERA) study. Am Heart J 2009;157:680-7. doi:10.1016/j.ahj.2008.12.013

4 Beygui F, Silvain J, Pena A, et al. Usefulness of biomarker strategy to improve GRACE score's prediction performance in patients with non-ST-segment elevation acute coronary syndrome and low event rates. Am J Cardiol 2010;106:650-8.

doi:10.1016/j.amjcard.2010.04.019

5 Montalescot G, Drexler H, Gallo R, et al. Effect of irbesartan and enalapril in non-ST elevation acute coronary syndrome: results of the randomized, double-blind ARCHIPELAGO study. Eur Heart J 2009;30:2733-41. doi:10.1093/eurheartj/ehp301

6 Fraccarollo D, Galuppo P, Schraut S, et al. Immediate mineralocorticoid receptor blockade improves myocardial infarct healing by modulation of the inflammatory response. Hypertension 2008;51:905-14. doi:10.1161/HYPERTENSIONAHA.107.100941

7 Perrier E, Kerfant B-G, Lalevee N, et al. Mineralocorticoid receptor antagonism prevents the electrical remodeling that precedes cellular hypertrophy after myocardial infarction. Circulation 2004;110:776-83. doi:10.1161/01.CIR.0000138973.55605.38

8 Hayashi M, Tsutamoto T, Wada A, et al. Immediate administration of mineralocorticoid receptor antagonist spironolactone prevents post-infarct left ventricular remodeling associated with suppression of a marker of myocardial collagen synthesis in patients with first anterior acute myocardial infarction. Circulation 2003;107:2559-65.

doi:10.1161/01.CIR.0000068340.96506.0F

9 Di Pasquale P, Cannizzaro S, Scalzo S, et al. Effects of canrenoate plus angiotensinconverting enzyme inhibitors versus angiotensin-converting enzyme inhibitors alone on systolic and diastolic function in patients with acute anterior myocardial infarction. Am Heart J 2005;150:919. doi:10.1016/j.ahj.2005.03.032

10 Denis B, Machecourt J, Denis MC, et al. [Value of potassium canrenoate in the prevention of arrhythmia during the acute phase of myocardial infarction]. Arch Mal Coeur Vaiss 1979;72:1014-22. 
11 Beygui F, Labbé J-P, Cayla G, et al. Early mineralocorticoid receptor blockade in primary percutaneous coronary intervention for ST-elevation myocardial infarction is associated with a reduction of life-threatening ventricular arrhythmia. Int J Cardiol 2013;167:73-9.

doi:10.1016/j.ijcard.2011.11.076

12 Pitt B, Zannad F, Remme WJ, et al. The effect of spironolactone on morbidity and mortality in patients with severe heart failure. Randomized Aldactone Evaluation Study Investigators. $N$ Engl J Med 1999;341:709-17. doi:10.1056/NEJM199909023411001

13 Zannad F, McMurray JJV, Krum H, et al. Eplerenone in patients with systolic heart failure and mild symptoms. N Engl J Med 2011;364:11-21. doi:10.1056/NEJMoa1009492

14 Pitt B, Remme W, Zannad F, et al. Eplerenone, a selective aldosterone blocker, in patients with left ventricular dysfunction after myocardial infarction. N Engl J Med 2003;348:1309-21. doi:10.1056/NEJMoa030207

15 Montalescot G, Pitt B, Lopez de Sa E, et al. Early eplerenone treatment in patients with acute ST-elevation myocardial infarction without heart failure: the Randomized Double-Blind Reminder Study. Eur Heart J 2014;35:2295-302. doi:10.1093/eurheartj/ehu164

16 Beygui F, Cayla G, Roule V, et al. Early Aldosterone Blockade in Acute Myocardial Infarction: The ALBATROSS Randomized Clinical Trial. J Am Coll Cardiol 2016;67:1917-27. doi:10.1016/j.jacc.2016.02.033

17 Zipes DP, Camm AJ, Borggrefe M, et al. ACC/AHA/ESC 2006 guidelines for management of patients with ventricular arrhythmias and the prevention of sudden cardiac death: a report of the American College of Cardiology/American Heart Association Task Force and the European Society of Cardiology Committee for Practice Guidelines (Writing Committee to Develop Guidelines for Management of Patients With Ventricular Arrhythmias and the Prevention of Sudden Cardiac Death). J Am Coll Cardiol 2006;48:e247-346. doi:10.1016/j.jacc.2006.07.010

18 Fraccarollo D, Galuppo P, Schraut S, et al. Immediate mineralocorticoid receptor blockade improves myocardial infarct healing by modulation of the inflammatory response. Hypertension 2008;51:905-14. doi:10.1161/HYPERTENSIONAHA.107.100941

19 Mihailidou AS, Loan Le TY, Mardini M, et al. Glucocorticoids activate cardiac mineralocorticoid receptors during experimental myocardial infarction. Hypertension 2009;54:1306-12. doi:10.1161/HYPERTENSIONAHA.109.136242

20 Modena MG, Aveta P, Menozzi A, et al. Aldosterone inhibition limits collagen synthesis and progressive left ventricular enlargement after anterior myocardial infarction. Am Heart J 2001;141:41-6. doi:10.1067/mhj.2001.111258

21 McMurray JJV, Adamopoulos S, Anker SD, et al. ESC Guidelines for the diagnosis and treatment of acute and chronic heart failure 2012: The Task Force for the Diagnosis and Treatment of Acute and Chronic Heart Failure 2012 of the European Society of Cardiology. Developed in collaboration with the Heart Failure Association (HFA) of the ESC. Eur Heart J 2012;33:1787-847. doi:10.1093/eurheartj/ehs104 
22 O'Gara PT, Kushner FG, Ascheim DD, et al. 2013 ACCF/AHA guideline for the management of ST-elevation myocardial infarction: a report of the American College of Cardiology Foundation/American Heart Association Task Force on Practice Guidelines. J Am Coll Cardiol 2013;61:e78-140. doi:10.1016/j.jacc.2012.11.019

23 Amsterdam EA, Wenger NK, Brindis RG, et al. 2014 AHA/ACC Guideline for the Management of Patients with Non-ST-Elevation Acute Coronary Syndromes: a report of the American College of Cardiology/American Heart Association Task Force on Practice Guidelines. J Am Coll Cardiol 2014;64:e139-228. doi:10.1016/j.jacc.2014.09.017

24 Adamopoulos C, Ahmed A, Fay R, et al. Timing of eplerenone initiation and outcomes in patients with heart failure after acute myocardial infarction complicated by left ventricular systolic dysfunction: insights from the EPHESUS trial. Eur J Heart Fail 2009;11:1099-105. doi:10.1093/eurjhf/hfp136

25 Sigurdsson A, Held P, Swedberg K. Short- and long-term neurohormonal activation following acute myocardial infarction. Am Heart J 1993;126:1068-76.

26 Foy SG, Crozier IG, Richards AM, et al. Neurohormonal changes after acute myocardial infarction. Relationships with haemodynamic indices and effects of ACE inhibition. Eur Heart J 1995;16:770-8.

27 Bossard M, Binbraik Y, Beygui F, et al. Mineralocorticoid receptor antagonists in patients with acute myocardial infarction - A systematic review and meta-analysis of randomized trials. Am Heart J 2018;195:60-9. doi:10.1016/j.ahj.2017.09.010

28 Mehta RH, Starr AZ, Lopes RD, et al. Incidence of and outcomes associated with ventricular tachycardia or fibrillation in patients undergoing primary percutaneous coronary intervention. JAMA 2009;301:1779-89. doi:10.1001/jama.2009.600

29 Cardiac Arrhythmia Suppression Trial (CAST) Investigators. Preliminary report: effect of encainide and flecainide on mortality in a randomized trial of arrhythmia suppression after myocardial infarction. $N$ Engl J Med 1989;321:406-12. doi:10.1056/NEJM198908103210629

30 Taniguchi T, Shiomi H, Morimoto T, et al. Incidence and Prognostic Impact of Heart Failure Hospitalization During Follow-Up After Primary Percutaneous Coronary Intervention in ST-Segment Elevation Myocardial Infarction. Am J Cardiol 2017;119:1729-39.

doi:10.1016/j.amjcard.2017.03.013 


\section{Figure Legends}

Figure 1. Kaplan-Meier cumulative hazard curves for death

Figure 2. Kaplan-Meier cumulative hazard curves for death or resuscitated sudden death Supplementary Figure. Subgroup analysis
A. Death
B. Death or Resuscitated Sudden Death

ACEi: angiotensin conversion enzyme inhibitor; ARB: angiotensin receptor ablockers, PCI:

percutaneous coronary intervention, LVEF: left ventricular ejection fraction; eGFR: estimated glomerular filtration rate; BMI: body mass index 